REZENSIONEN

\section{Leben regulieren? Reflexionen zu den Risiken der Synthetischen Biologie}

\author{
B. Giese, C. Pade, H. Wigger, A. von \\ Gleich (Hg.): Synthetic Biology - \\ Character and Impact. Heidelberg u. a.: \\ Springer 2015, 273 S., ISBN 978-3-319- \\ 02782-1, Euro 106,99
}

\section{Rezension von Julia Diekämper und Anja Hümpel, Berlin-Brandenburgische Akademie der Wissenschaften, Berlin}

Synthetische Biologie ist ein heißes Eisen - einschlägige Publikationen, die sich in den letzten Jahren den vielschichtigen im Kontext dieser noch jungen Disziplin entstehenden Fragen widmeten, belegen dies eindrücklich. Dem stetig wiederholten Versprechen der Forschungsgemeinde, diese Aushandlungen interdisziplinär zu führen, schließt sich der von Bernd Giese, Christian Pade, Henning Wigger und Arnim von Gleich herausgegebene Sammelband an. Entstanden aus einer Vortragsreihe „Technology Assessment of Synthetic Biology" an der Universität Bremen erscheint es in der vom Springer Verlag initiierten Reihe „Risk Engineering“, die die Technik zum Anlass nimmt und aus verschiedenen Perspektiven auf das Thema Risiko schaut. Damit ist auch ein zentrales Anliegen des Bandes verbunden: In 13 Kapiteln soll nicht weniger geleistet werden, als das Forschungsfeld Synthetische Biologie zu charakterisieren, Einsatzmöglichkeiten abzuschätzen, Risiken für Mensch und Umwelt zu diskutieren sowie rechtliche Rahmenbedingungen und Fragen nach dem Topos der Verantwortung zu analysieren.

\section{Technologisierung der Biologie}

Synthetische Biologie war und ist ein Begriff, der eine große Spannbreite an Forschungsansätzen vereint. Entsprechende Definitionsvorschläge sind so vielstimmig wie Verortungen und Hi- erarchisierungen dessen, was unter dem Begriff genau zu subsumieren sei. Zentral ist die Frage, wie neu oder anders die Synthetische Biologie sei und was sie konkret leiste. Der erste Teil des Sammelbandes (Kap. 1-4) bietet hierzu eine Fülle an Thesen und Theorien, wie sich das Synthetische mit dem Biologischen möglicherweise verbinden lässt: Aus grundlagenforschender Biologie wird anwendungsorientierte Technowissenschaft.

Jan C. Schmidt stellt die Synthetische Biologie als Schlüsseltechnologie der Spätmoderne („late-modern technology“) vor. Populäre Definitionen des Feldes greifen jedoch seines Erachtens zu kurz. Schmidt diagnostiziert einen Umbruch für unser Verständnis von Technologie, der über die Synthetische Biologie hinaus zu gehen vermag (,epochal break is occurring in our understanding of technology", S. 25). Er begründet seine Ausführungen vor einem theoretischen Netz, das von Aristoteles bis Kant gespannt ist, mit der Notwendigkeit einer prospektiven TAAnalyse, um negativen Effekten vorzubeugen, die sich aus dem Konflikt von Hype und Hybris der Synthetischen Biologie im öffentlichen Diskurs ergeben könnten.

Die Vielstimmigkeit philosophischer Diskurse illustriert beispielhaft Alfred Nordmann anlässlich der Synthetischen Biologie, indem er die Konfliktlinien zwischen klassischen Ingenieursprinzipien und der Funktionalität biologischer Prozesse nachvollzieht und feststellt, diese lassen sich nicht einfach auflösen, sie verlangen vielmehr eine neue Art der Betrachtung (,,antagonism between epistemic ideals", S. 55). Er diagnostiziert einen Wandel der Erkenntnisproduktion, den er festmacht an einer neuen Priorisierung der technischen Gestaltung lebender Systeme. In seinem Text stellt er Ingenieurbiologie und Erkenntnistheorie in einen Zusammenhang: Wie müssen biologische Systeme verstanden werden, um technische Kontrolle zu erhalten? Dabei setzt er drei Begriffe ins Zentrum, die im Diskurs heterogen rezipiert werden: Verständnis, Rationalität und Design.

Zwei Lösungsansätze hierfür präsentiert Michael Bölker: Einerseits die Anwendung von Ingenieursprinzipien, andererseits die Orthogonalisierung (d. h. die Unterbindung von Interak- 
tion zwischen Zellkomponenten, Prozessen, Zellen etc.). Dabei diskutiert er, die aus den Ansätzen ableitbaren Sicherheitsaspekte. Er gelangt zu dem Fazit, dass vereinfachte konstruierte Systeme die höchste Sicherheit versprechen. Mit mehr Komplexität allerdings steige auch die Unsicherheit und möglicherweise das Risiko.

Christian Pade und Kollegen bieten ein umfangreiches Konzept zur Charakterisierung und Risikoabschätzung des unübersichtlichen Feldes der Synthetischen Biologie an, das auf dem Begriff der Funktionalität (,functionalities“) aufbaut. Ihr Fokus liegt vor allem auf den neuartigen biologischen Eigenschaften, die durch synthetische Ansätze denkbar sind. Die Skizze möglicher Risikoszenarien, die sich aus einzelnen Anwendungen ergeben könnten, erfolgt hier ohne Bewertung. Dem Leser bleibt es überlassen, hieraus gute Gründe zu destillieren. Hierfür wäre eine Hierarchisierung hilfreich gewesen.

\section{Making life better. Aufbruch zu neuen Welten?}

Mit seinem Kapitel leitet Gerd Klöck die Betrachtung der praktischen Anwendung im Sammelband ein (Kap. 5-8). Dabei wird der Status quo der industriellen Biotechnologie vorgestellt, die gentechnisch veränderte Mikroorganismen für die Produktion von z. B. Vitaminen oder Enzymen nutzt. Um aber die Abkehr von fossilen Brennstoffen bei Erschließung neuer nachwachsender Rohstoffquellen zu gewährleisten, sieht Klöck die Notwendigkeit für innovative Ansätze, die die technischen Beschränkungen gegenwärtiger Produktionsverfahren (biotechnologischer wie chemischer) zu überwinden vermögen. Genau hier liegt für ihn das industrielle Anwendungspotenzial der Synthetischen Biologie.

Zwei prominente Forschungsziele der Synthetischen Biologie - Biosensoren und der biokatalytische Abbau von Biomasse - greifen vertiefend Christopher E. French und Kollegen auf. Es gilt, die richtigen biologischen Bauteile, Module und Kassetten zu generieren, zusammenzusetzen und im Sinne eines ,combinatorial genetic engineering“" zu testen (S. 130). Damit lassen sich, so die Autoren, innovative Lösungen finden, die über die bisherigen gentechnologischen Möglichkeiten hinausgehen.

Auch Stefan M. Schiller stellt die Modularität intrazellulärer Vorgänge in den Vordergrund: Es bedarf seines Erachtens eines Katalogs funktionaler Bausteine im Sinne einer ,synthetic biolog-toolbox" (S. 140). Nicht-kanonische Aminosäuren, die im Gegensatz zu den mehr als zwanzig natürlichen Aminosäuren nicht im universellen genetischen Code festgelegt sind, erlauben eine Fülle an Proteinmodifikationen, die unterschiedlichste Eigenschaften und Strukturen zu generieren vermögen. Der Beitrag lotet angesichts visionärer Vorstellungen mögliche Entwicklungslinien der Disziplin aus.

Dementgegen dringt Antoine Danchin darauf, das Leben wieder in seiner komplexen Gesamtheit in den Blick zu nehmen, wenn es darum geht auf beliebige Zwecke zu programmierende synthetische Zellen (,chassis“) zu erzeugen. Er lädt Lesende zur Auseinandersetzung mit den Minimalanforderungen des Lebens ein, die bereits seit Jahrzehnten Gegenstand der Forschung sind: Welche Gene, welche Eigenschaften sind zentral? Er warnt vor einem zu einseitigen Fokus auf Sequenzen und Strukturen. Somit sind für ihn die an den Bottom-up-Ansatz gebundenen Prämissen überprüfungswürdig (,the standard 'Rosetta stone' stance, based on the bottom-up approach that uses sequence-based comparative genomics to identify conserved genes, is doomed to fail at least if taken without a grain of salt", S. 160). Das Verständnis der Funktion muss im Vordergrund stehen und zwar in seiner ganzen Komplexität über die Lebenszeit eines Organismus.

\section{Risk-Reducing Strategies}

Mit der (zukünftigen) Umsetzung der verschiedenen Forschungsansätze der Synthetischen Biologie sind die Fragen nach der biologischen Sicherheit und der notwendigen Governance eng verknüpft. Der dritte Teil des Sammelbandes (Kap. 9-13) reflektiert konsequent über Gefährdungspotenziale und geeignete Regularien.

Bernd Giese und Arnim von Gleich plädieren in ihrem Beitrag für eine frühe Begleitung der Synthetischen Biologie (S. 175). Der Appell deckt sich mit der allgemeinen Tendenz des Bandes. 
Aufdeckung und Assessment von Risikogefahren seien aktuell Hauptanliegen der entsprechenden Forschung. Risiken wie Nutzen hängen für sie nicht nur von eben jener Funktionalität ab, sondern auch vom spezifischen Kontext und der intendierten Anwendung. Einer Risikoeinschätzung kommt man dabei ihres Erachtens über das jeweilige Verhältnis zu bestimmten Fähigkeiten näher.

Broder Breckling und Gunther Schmidt nehmen die Parallelität der Gentechnik (,genetic engineering") zur Synthetischen Biologie in ihrem Beitrag in den Blick, um hieraus einen Handlungsbedarf auszuloten. Sie diskutieren dabei Risikoüberlegungen auf unterschiedlichen Ebenen: molekular, zellulär, auf der Ebene der Organismen, auf der Ebene der Population, des Ökosystems, der Umwelt. Dabei kommen sie zu dem Schluss, dass Risikomanagement notwendigerweise auf dem molekularen Niveau beginnen muss (S. 205). Zwar sei es möglich, so ihr Fazit, Parallelen zwischen beiden Debatten zu ziehen (S. 208), dennoch sei es geraten, die unterschiedlichen Ebenen bei der Risikoanalyse zu unterscheiden.

Einen Vergleich zwischen Synthetischer Biologie und Gentechnik macht auch Gerd Winter in seinem Beitrag nutzbar. Vor einem juristischen Horizont lotet er aus, inwiefern angesichts neuer technischer Verfahren (europäischer) Handlungsbedarf bestehe. Er gelangt zur Schlussfolgerung, dass entgegen offizieller Statements das existierende Regelwerk nicht für die Synthetische Biologie Anwendung finden kann. Es brauche vielmehr eine Ausweitung des Umfangs und eine Verbesserung des Risikoassessments bestehender GMO-Regelungen. Alternativ sei es angezeigt, einen neuen Zugang für eine Betrachtung zu wählen, der Biotechnologie in Gänze in den Blick nimmt.

Eine andere Perspektive wählt Joachim Boldt, der die Synthetische Biologie auf ihren utopischen/dystopischen Gehalt befragt. Die utopische Vision bestehe dabei genauer darin, dass in der Synthetischen Biologie das Potenzial gesehen werde, eine Gesellschaft zu begründen, die im Einklang mit der Natur lebt, wohingegen die Dystopiker hierin eine Zerstörung eben jenes Verhältnisses befürchten. Hieran zeigt Boldt einen kommunikativen und einen instrumentellen
Modus der Handlung auf. Er schlägt eine Lesart der ethischen Betrachtung der Synthetischen Biologie unter Bezugnahme auf Habermas' Theorie des kommunikativen Handelns vor. Eine kantianische Auslegung bringt ihn zu dem Schluss, dass kommunikative Handlung zu einer biozentristischen ethischen Haltung führen kann, die nicht utilitaristisch sei (S. 243). Angewendet auf die Synthetische Biologie unterstreicht er, dass sich selbstverständlich zwischen den beiden Modi eine variable Grenze befinde.

Armin Grunwalds Aufsatz bildet den Abschluss des Sammelbandes und bietet Einblick in die komplexen Fragen der Verantwortung, die die Synthetische Biologie als neue Technowissenschaft zeitigt. Er votiert dabei für eine präventive ethische Auseinandersetzung und macht zudem unmissverständlich deutlich, dass keine Disziplin in der Konfrontation mit den durch die Synthetische Biologie aufgeworfenen Fragen alleine reüssieren könne. Er plädiert daher für einen umfassenden interdisziplinären Diskurs, der über die rein wissenschaftliche Sphäre hinausgreift und alle Akteure, Stakeholder und nicht zuletzt die allgemeine Öffentlichkeit einbezieht.

\section{Neue Impulse für ein heißes Thema}

Der Sammelband fügt dem vielstimmigen Diskurs ganz offensichtlich etwas hinzu. Und das sowohl inhaltlich als auch strukturell. Das Spezifikum des Bandes besteht darin, dass er Klarheit und Ordnung in das unübersichtliche Feld der Synthetischen Biologie zu bringen verspricht. Das ist insofern neu, als dass es zuvor in den entsprechenden begleitenden Debatten mäandernde Tendenzen gab, denen die Beiträge hier mehrheitlich thesenstark entgegentreten. Vergleicht man etwa hier getroffene Aussagen mit denen der Stellungnahme von DFG, acatech und Leopoldina von 2009, dann scheint auf den ersten Blick inzwischen ein stabiles Fundament geschaffen. Auf den zweiten gilt es allerdings zu fragen, ob die so geschaffene Sicherheit tatsächlich trägt. Schließlich geht es auch in anderen Bereichen der Biowissenschaften um immer mehr fachübergreifende Zusammenarbeit und zunehmende Anwendungsorientierung. Der Anspruch an den großen Umbruch der Gentechnologie und neuar- 
tige Technowissenschaft wird gerade im Anwendungsteil nicht konsequent eingelöst. Fraglich - das allerdings gilt es nicht explizit für dieses Buch zu diskutieren, sondern ist zumindest ein weiteres Charakteristikum der sich um die Synthetische Biologie rankenden Auseinandersetzungen - ist, wen genau die Konzeptionalisten eines derart facettenreichen und fachspezifischen Diskurses vor Augen haben. Die vielbeschworene allgemeine Öffentlichkeit mag es nicht sein.

$《 》$

\section{Von der Wissenschaftsforschung zur Wissenschaftspolitik}

E. Grande, D. Jansen, O. Jarren, A. Rip,
U. Schimank, P. Weingart (Hg.): Neue
Governance der Wissenschaft. Reorgani-
sation - externe Anforderungen - Mediali-
sierung. Bielefeld: transcript 2013, 374 S.,
ISBN 978-3-8376-2272-0, Euro 32,80

Rezension von David Kaldewey, Abteilung Wissenschaftsforschung, Universität Bonn

\section{Wissenschaft und Politik}

Zur Charakterisierung des Verhältnisses von Wissenschaft und Politik werden seit Harvey Brooks (1968) zwei Einflussrichtungen unterschieden: Auf der einen Seite geht es um die Verwendung wissenschaftlichen Wissens in politischen Entscheidungsprozessen, vermittelt etwa durch wissenschaftliche Politikberatung (science for policy), auf der anderen Seite um die politische Aufgabe der Aufrechterhaltung eines funktionierenden Wissenschaftssystems, etwa durch Finanzierung von Institutionen oder Forschungsprogrammen (policy for science). Dass diese Unterscheidung nicht immer scharf zu ziehen ist, hat Brooks nie bestritten, und lässt sich auch an einer Beobachtung von Rudolf Stichweh (2013) illustrieren, der zufolge Wissenschaftspolitik im zweiten Sinne häufig von Wissenschaftlern betrieben wird, die dauerhaft oder temporär die Rolle wissenschaftlicher Politikberater übernehmen, und in dieser neuen Rolle die Wissenschaft politisch zu steuern suchen. Ein weiterer Sonderfall liegt vor, wenn die politikberatende Rolle nicht von ,normalen' Wissenschaftlern übernommen wird, die im Verlauf ihrer Karriere mit Seitenwechseln experimentieren, sondern von Wissenschaftsforschern, die sich thematisch auf die Wissenschaft als soziales System und auf deren Verhältnis zur gesellschaftlichen Umwelt spezialisiert haben. Tatsächlich zeichnen sich insbesondere die Science and Technology Studies (STS) durch einen entsprechenden Trend zur Politisierung aus: Steve Fuller (2007) etwa charakterisiert die STS als „political player“ und Sergio Sismondo (2008) skizziert in diesem Sinne ein ,engaged program“, das sich neben der epistemischen Durchdringung des Gegenstandes auch politischen Werten verpflichtet weiß.

Diese für die Identitätsarbeit der STS konstitutive Politisierung ist eine hilfreiche Folie zur Interpretation der in den letzten Jahren auch in Deutschland engagierter geführten Diskussion über das Verhältnis von Wissenschaftsforschung und Politik. Der von Edgar Grande et al. herausgegebene Sammelband „Neue Governance der Wissenschaft" lädt in diesem Zusammenhang dazu ein, anhand einer Reihe von Forschungsprojekten zu eruieren, wie die deutsche Wissenschaftsforschung - die sich bislang im Vergleich zu den dominanten Strömungen der STS durch ein „distanzierteres“ Verhältnis zu ihrem Forschungsgegenstand ausgezeichnet hatte - mit der Frage der politischen Relevanz umgeht.

\section{Die Rolle der BMBF-Programmförderung}

Die in dem Band präsentierten Projekte sind im Rahmen einer breit angelegten Förderinitiative des Bundesministeriums für Bildung und Forschung (BMBF 2008) durchgeführt worden. Hintergrund ist ein bereits 2001 aufgelegtes Programm, das darauf zielte, die in Deutschland - zumal im Vergleich mit anderen europäischen Ländern - nur schwach institutionalisierte Wissenschaftsforschung durch gezielte Schwerpunktbildung besser aufzustellen. Lanciert wurde daraufhin die zweiphasige, mit einem Volumen von rund 15 Mio. Euro ausgestatte Förderinitiative „Forschung zum Verhältnis von Wissenschaft, Politik und Gesellschaft“. In der 